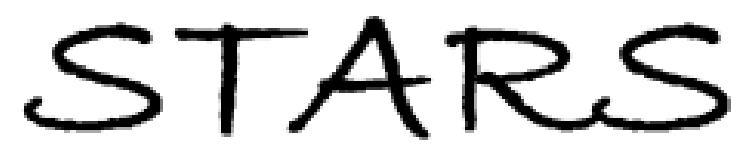

University of Central Florida

STARS

\title{
Power-law eigenvalue density, scaling, and critical random-matrix ensembles
}

\author{
K. A. Muttalib \\ Mourand E. H. Ismail \\ University of Central Florida
}

Find similar works at: https://stars.library.ucf.edu/facultybib2000 University of Central Florida Libraries http://library.ucf.edu

This Article is brought to you for free and open access by the Faculty Bibliography at STARS. It has been accepted for inclusion in Faculty Bibliography 2000s by an authorized administrator of STARS. For more information, please contact STARS@ucf.edu.

\section{Recommended Citation}

Muttalib, K. A. and Ismail, Mourand E. H., "Power-law eigenvalue density, scaling, and critical randommatrix ensembles" (2007). Faculty Bibliography 2000s. 7458.

https://stars.library.ucf.edu/facultybib2000/7458

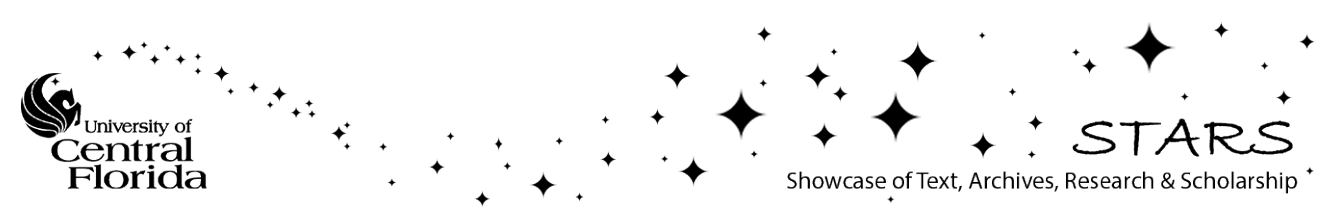




\title{
Power-law eigenvalue density, scaling, and critical random-matrix ensembles
}

\author{
K. A. Muttalib* \\ Department of Physics, University of Florida, P.O. Box 118440, Gainesville, Florida 32611-8440, USA
}

Mourad E. H. Ismail ${ }^{\dagger}$

Department of Mathematics, University of Central Florida, Orlando, Florida 32816, USA

(Received 18 July 2007; published 8 November 2007)

\begin{abstract}
We consider a class of rotationally invariant unitary random matrix ensembles where the eigenvalue density falls off as an inverse power law. Under a scaling appropriate for such power-law densities (different from the scaling required in Gaussian random matrix ensembles), we calculate exactly the two-level kernel that determines all eigenvalue correlations. We show that such ensembles belong to the class of critical ensembles.
\end{abstract}

DOI: 10.1103/PhysRevE.76.051105

\section{INTRODUCTION}

Gaussian random matrix ensembles, introduced by Wigner and Dyson [1], have been studied extensively over half a century in the context of nuclear physics, atomic and molecular physics, condensed matter physics, as well as particle physics [2]. The wide applicability results from the universal properties of the correlation functions of the eigenvalues, once they are appropriately scaled. Thus, e.g., the correlation functions of the eigenvalues $x$ of an ensemble of $N \times N$ Hermitian matrices become, after proper scaling, independent of the size of the matrix or of the details of the microscopic distribution from which the matrix elements are drawn. For a Gaussian distribution, the density is a semicircle given by $\sigma(x)=\sqrt{2 N-x^{2}} / \pi$; this requires, for universality, the double scaling limit

$$
N \rightarrow \infty, \quad x \rightarrow 0, \quad u \equiv x \sqrt{N} \quad \text { finite }
$$

such that the density $\bar{\sigma}(u)=$ const near the origin. This particular double scaling is required for all Gaussian random matrix ensembles of different symmetry classes [3] in order to have a universal large $N$ limit. In particular, for Gaussian unitary ensembles, after a second trivial scaling $\zeta=\sqrt{2} u / \pi$ such that $\widetilde{\sigma}(\zeta)=1$, it leads to the universal two-level kernel

$$
K^{G}(\zeta, \eta)=\frac{\sin [\pi(\zeta-\eta)]}{\pi(\zeta-\eta)}
$$

independent of $N$, which gives rise to universal eigenvalue correlations like the nearest-neighbor spacing distribution or the number variance, commonly known as the Wigner distributions.

Note that in the above double scaling limit, one is always restricted to the eigenvalues far from the tails of the density. This is a highly nontrivial feature of Gaussian random matrix ensembles. Indeed, the eigenvalue correlations for Gaussian ensembles are deeply related to the properties of Hermite polynomials [3], and the above scaling is dictated by the asymptotic properties of Hermite polynomials of order $N$ and $\operatorname{argument} x$ in the large $N$ limit for small $x$. Since Hermite

\footnotetext{
*muttalib@phys.ufl.edu

†ismail@math.ucf.edu
}

PACS number(s): 05.40.-a, 05.60.-k, 05.90.+m

polynomials have a different asymptotic behavior for large $x$ in the large $N$ limit [4], a different scaling is dictated for the Gaussian ensembles near the edge of the semi-circle spectrum. Here universality is recovered after a shift $x^{\prime}=x$ $-\sqrt{2 N+1}$, and a different double scaling limit near the new origin, namely $N \rightarrow \infty, x^{\prime} \rightarrow 0$, with $u \equiv x^{\prime} N^{1 / 6}$ remaining finite [5]. In this case one gets the so-called Airy kernel and the density decreases exponentially for large $u$.

In a sense, the universality of the Gaussian random matrix ensembles is a result of an underlying central limit theorem, generalized to matrices [6]. Indeed, the universality requires that the eigenvalues are confined "strongly enough" such that the fluctuations remain bounded. For example, if the probability density $P(H)$ for an $N \times N$ random Hamiltonian $H$ is given by

$$
P(H) \propto \exp [-\beta \operatorname{Tr} V(H)]
$$

then in a more general situation where the confining potential $V(H)$ is not a Gaussian, the density may not be the same near the origin. Even then, one can "unfold" the spectrum by choosing a variable in which the density is unity, and as long as the confining potential is "strong enough," the double scaling given by Eq. (1) always leads to the same two-level kernel and therefore the same universal correlations. This is because for any such rotationally invariant ensemble described by a confining potential, the two-level kernel can be described in terms of polynomials orthogonal with respect to a Freud-type weight function $e^{-V(x)}$, with polynomial $V$, [just as the Hermite polynomials arise for Gaussian ensembles where the potential is $V(x)=x^{2}$ ] [3]. Again, the eigenvalue correlations are deeply related to the properties of those orthogonal polynomials. However, all Freud-type orthogonal polynomials have qualitatively similar asymptotic behavior in the large $N$ limit. Therefore the same double scaling limit leads to universality for all such different confining potentials. Conversely, an arbitrary scaling (with, e.g., an arbitrary power law for $N$ ) does not lead to a universal $N$-independent kernel for rotationally invariant random matrix ensembles associated with the classical orthogonal polynomials.

On the other hand, it is known that the universality does break down if the confinement potential $V(H)$ grows extremely slowly with $H[7]$, namely 


$$
V(H)=\frac{1}{\gamma} \ln ^{2} H, \quad H \gg 1 .
$$

This leads to the so-called "critical" ensembles [8], characterized by a one-parameter generalization of the Gaussian two-level kernel, given by [7,9]

$$
K^{C}(\zeta, \eta ; \gamma)=\frac{\gamma}{2 \pi} \frac{\sin [\pi(\zeta-\eta)]}{\sinh [(\zeta-\eta) \gamma / 2]}, \quad \gamma \ll 2 \pi^{2} .
$$

The eigenvalue correlations for such systems for various values of the parameter $\gamma$ have been studied in the context of the Anderson transition in disordered quantum conductors [10]. The density of the eigenvalues with the weak confinement potential (4) turns out to be a constant independent of $N$ in the $N \rightarrow \infty$ limit, and the scaled variables are simply $\zeta$ $=x / \gamma$. Note that the critical ensembles tend to the Gaussian ensembles in the limit $\gamma \rightarrow 0$, in which case one is again restricted to the correlations of the eigenvalues near the origin. However, the scaling does not involve $N$. This difference in the scaling behavior is the reason why critical ensembles could not be studied using the classical orthogonal polynomials.

A second case where a different scaling beyond the scope of the Freud-type orthogonal polynomials is required is when the density of the eigenvalues $x$ falls off as a power law for large $x$. This indeed happens for a large class of random matrix ensembles of recent interest, relevant for, e.g., finance or scale free systems that require considering power-law distributions with fat tails $[11,12]$. In a pioneering study, Cizeau and Bouchaud (CB) [6] introduced the so-called "Lévy matrix ensembles" where the matrix elements are drawn from a power-law distribution according to $P\left(H_{i j}\right)$ with

$$
P\left(H_{i j}\right) \sim \frac{H_{0}^{\mu}}{\left|H_{i j}\right|^{1+\mu}}, \quad H_{i j} \gg 1 .
$$

Here $H_{0}$ is of order $H_{i j}$ and the parameter $\mu \geq 0$. The eigenvalue density in this case was shown to fall off with the same exponent, as $1 / x^{1+\mu}$. In particular for $0<\mu<1$, the distribution has diverging variance and hence the underlying central limit theorem must now be modified according to the theorems of Lévy and Gnedenko [13]. Indeed, several numerical works have shown $[13,14]$ that the universality of the Gaussian ensembles does break down when the density of eigenvalues follows a power-law with exponent less than 2 , the signature of which is apparent in the numerically obtained nearest-neighbor spacing distribution and the number variance. The eigenvalue densities for certain Lévy ensembles have been obtained analytically [12], but so far it has not been possible to evaluate the two-level kernel which gives the two or higher level eigenvalue correlations. Since the universality requires "unfolding" where the density is made uniform, it is obviously of more interest to obtain the twolevel kernel for a Lévy-like ensemble, specially where the microscopic distribution leads to a power-law eigenvalue density which falls off with an exponent less than 2 .

Clearly, if the density of eigenvalues falls off with a power law and we are interested in the properties of the large eigenvalues in the tails, any possible universality of the as- sociated random matrix ensembles cannot be expected in the same double scaling limit used for the Gaussian ensembles, either at the origin or near the semicircle edge. In [6], CB proposed a double scaling limit

$$
N \rightarrow \infty, \quad x \rightarrow \infty, \quad u \equiv x / N^{\alpha} \quad \text { finite },
$$

where $\alpha>0$ is related to the power-law exponent, in which the Lévy ensembles might become universal. We will call this the CB scaling. As mentioned above, this scaling cannot be obtained naturally within the scheme of classical or Freud-type orthogonal polynomials. This means that the most powerful approach to study eigenvalue correlations of random matrix ensembles, namely the method of orthogonal polynomials [3], seems to be inapplicable for Lévy-type random matrices. It is for this reason that progress has been slow in detailed analytic studies of eigenvalue correlations of random matrices with power-law densities.

On the other hand, although in most studies it has been implicitly assumed that a power-law density of eigenvalues requires choosing matrix elements from a power-law distribution of the type (6), we find that at least for the inverse power law with exponent 1 , this is not the case. Indeed, we find that a weakly confined log-squared potential that gives rise to the critical ensemble with a constant density for an $N$-independent scaling also gives rise to a inverse power-law density under the CB scaling. It turns out that the weakly confined log-squared potential is exactly solvable even in the new CB scaling regime and the two-level kernel in this scaling regime has a well-defined limit as $N \rightarrow \infty$.

\section{MODEL}

In the present work we use the solvability of the log squared potential to obtain analytically, exactly, and explicitly, the two-level kernel for a random matrix ensemble with density $\sigma(x) \propto 1 / x$ in the $\mathrm{CB}$ double scaling limit given in Eq. (7) where $\alpha$ is an arbitrary positive parameter. Specifically, we use the model characterized by the confinement potential

$$
V(x \mid q)=\frac{2}{|\ln q|}\left[\ln \left(x+\sqrt{1+x^{2}}\right)\right]^{2},
$$

where $q$ is a parameter, $0<q \leq 1$. The joint probability distribution of the $N$ eigenvalues can then be written as [3]

$$
P(\{x\})=C_{N}(q) \prod_{i<j}^{N}\left(x_{i}-x_{j}\right)^{2} \prod_{i=1}^{N} e^{-V(x \mid q)},
$$

where $C_{N}(q)$ is a constant and the product $\Pi_{i<j}^{N}\left(x_{i}-x_{j}\right)^{2}$ is the standard Vandermonde factor for unitary ensembles. The orthogonal polynomials corresponding to the weight function

$$
w_{H}(x \mid q)=q^{1 / 8} \sqrt{\frac{-2}{\pi \ln q}} e^{-V(x \mid q)}
$$

for the above confining potential, in terms of which the twolevel kernel can be written down exactly, are the IsmailMasson $q^{-1}$-Hermite polynomials [15] first considered in the context of random matrices in [7], where only an 
$\mathrm{N}$-independent scaling was considered. The reason for the choice of the potential (8) is that a very general asymptotic relation for the corresponding Ismail-Masson polynomials has been obtained recently [16], such that the new CB scaling can be implemented explicitly without compromising the solvability of the model. In particular, for

$$
x_{n}(t, u)=\frac{1}{2}\left(q^{-n t} u-q^{n t} / u\right)
$$

and $0<t<1 / 2[17]$ it has been shown that the normalized Ismail-Masson polynomials $\widetilde{h}_{n}\left(x_{n} \mid q\right)$ has the large $n$ limit given by

$$
\sqrt{w_{H}\left(x_{n}\right)} \tilde{h}_{n}\left(x_{n}\right)=\frac{\sqrt{w_{H}(\sinh \ln u \mid q)} u^{2 a}}{(-1)^{\lfloor m / 2\rfloor}(q ; q)_{\infty} \sqrt{(q ; q)}} q^{n / 4} q^{a^{2}} \Theta\left(-u^{2} q^{2 a}, q\right) .
$$

Here $(q ; q)_{n}=\Pi_{0}^{\infty}\left(1-q^{n}\right), m$ is an integer, and $0 \leq \lambda<1$ has been defined by the relation $m+\lambda=n(1-2 t)$. We have also defined $2 a=\chi(m)+\lambda$ where $\chi(m)$ is $0(1)$ for $m=$ even(odd). The function

$$
\Theta(z, q)=\sum_{n=-\infty}^{\infty} q^{n^{2}} z^{n}
$$

is defined for $z \neq 0$ for any complex $z$.

Let us define

$$
q \equiv e^{-\gamma}, \quad \gamma \geq 0 .
$$

Note that for $N \rightarrow \infty$ and large $u$, we can choose the parameters $\gamma$ and $t$ to satisfy the CB scaling for arbitrary $\alpha$. The choice

$$
\gamma N t=\alpha \ln N,
$$

when used in Eq. (11), gives precisely the CB scaling $u$ $=x / N^{\alpha}$. Note that while for finite $0<t<1 / 2$ the above condition requires $\gamma=2 \alpha \ln (N) / N \rightarrow 0$ or equivalently $q \rightarrow 1$, we are free to choose $t \sim \ln (N) / N$ such that the scaling relation can be satisfied for finite $\gamma$. In the following, we will outline the derivation of the two-level kernel for $\gamma \ll \pi^{2}$.

\section{TWO-LEVEL KERNEL}

The two-level Ismail-Masson kernel defined in terms of the orthonormal polynomials $\widetilde{h}_{n}(x \mid q)$ is given by $K_{N}(x, y)$ $=\sqrt{w_{H}\left(x_{N}\right) w_{H}\left(y_{N}\right)} \sum_{k=0}^{N-1} \widetilde{h}_{k}(x \mid q) \widetilde{h}_{k}(y \mid q)$. For large $N$ it can be written, using the Christoffel-Darbeau formula, as

$$
\begin{aligned}
K_{N}(x, y)= & \sqrt{w_{H}\left(x_{N}\right) w_{H}\left(y_{N}\right)} \\
& \times \frac{k_{N}}{k_{N-1}} \frac{\widetilde{h}_{N}\left(x_{N} \mid q\right) \widetilde{h}_{N-1}\left(y_{N} \mid q\right)-\widetilde{h}_{N}\left(y_{N} \mid q\right) \widetilde{h}_{N-1}\left(x_{N} \mid q\right)}{x-y},
\end{aligned}
$$

where $k_{N}$ is the coefficient of the term $x^{N}$ in the polynomial of order $N$. In Eq. (16) we can replace $N$ by $N-1$ to obtain the asymptotic expression for the normalized polynomials
$\tilde{h}_{N-1}\left(x_{N-1} \mid q\right)$. However, the Christoffel-Darbeaux formula involves $\tilde{h}_{N-1}\left(x_{N} \mid q\right)$. Similarly, we need expression for the weight factor $w_{H}\left(x_{N-1} \mid q\right)$ in terms of $w_{H}\left(x_{N} \mid q\right)$. We obtain these results by exploiting the scaling relation

$$
x_{N-1}\left(t, q^{-t} u\right)=x_{N}(t, u),
$$

which follows from Eq. (11). This gives, in terms of the scaled variables $v=y / N^{\alpha}$,

$$
\begin{aligned}
\sqrt{w_{H}\left(y_{N}\right)} \tilde{h}_{N-1}\left(y_{N}\right)= & \frac{\sqrt{w_{H}\left(\sinh \ln \left(q^{-t} v\right) \mid q\right)}}{(-1)^{\left\lfloor m^{\prime} / 2\right\rfloor}(q ; q)_{\infty} \sqrt{(q ; q)_{N-1}}} \\
& \times\left(q^{-t} v\right)^{2 b+2 t} q^{(N-1) / 4} q^{(b+t)^{2}} \Theta\left(-v^{2} q^{2 b}, q\right),
\end{aligned}
$$

where $m^{\prime}+\lambda^{\prime}=(N-1)(1-2 t)$ and we have defined $2 b$ $=\chi\left(m^{\prime}\right)+\lambda^{\prime}-2 t$.

The functions $\Theta(z, q)$ appearing in the above equations cannot be evaluated easily. However, for $q$ near 1 (or $\gamma$ $\ll \pi^{2}$ ) which is the region we would be interested in (this allows us to go to the Gaussian limit $q \rightarrow 1$ ), we can use the imaginary transformation $[18]$

$$
\Theta(w, q)=\sqrt{\frac{\pi}{\gamma}} e^{\ln { }^{2} w / 4 \gamma} \Theta\left(e^{\pi \ln w / i \gamma}, p\right) ; \quad p=e^{-\pi^{2} / \gamma} .
$$

In this case the series in powers of $p=e^{-\pi^{2} / \gamma}$ converges rapidly for $\gamma \ll \pi^{2}$ and can be approximated quite well by keeping only the dominant term. The transformation allows us to rewrite the kernel as

$$
\begin{aligned}
(x-y) K(x, y)= & A(q) e^{i \pi / \gamma \ln (u v)} \sum_{k_{1}, k_{2}} p^{k_{1}^{2}+k_{2}^{2}-k_{1}-k_{2}}\left(p z_{1}\right)^{k_{1}}\left(p z_{2}\right)^{k_{2}} \\
& \times\left[1-e^{-i 2 \pi(a-b)\left(k_{1}-k_{2}\right)}\right],
\end{aligned}
$$

where $p z_{1}=e^{i 2 \pi[a-1 / \gamma \ln u]}, p z_{2}=e^{i 2 \pi[b-1 / \gamma \ln v]}$, and $A(q)$ is a constant independent of $u, v$. We now make our first approximation, namely that we keep only the two terms $k_{1}=0, k_{2}$ $=1$ and $k_{1}=1, k_{2}=0$ in the double sum in Eq. (20). This neglects terms of order $p^{2}$ or higher powers of $p$. Using the fact that the product $(-1)^{[m / 2]+\left[m^{\prime} / 2\right\rfloor} \sin [\pi(a-b)]=1$ for all $a, b$, we finally obtain

$$
K(x, y)=K_{0} q^{n t} \frac{\sin \left[\frac{\pi}{\gamma} \ln \frac{u}{v}\right]}{u-v},
$$

where

$$
K_{0}=\sqrt{\frac{2}{\pi \gamma}} \frac{2 \pi}{\gamma} q^{-1 / 8} \frac{e^{-\pi^{2} / 2 \gamma}}{(q ; q)_{\infty}^{3}} .
$$

In order to estimate $K_{0}$, we use the Euler identity [19] $(q ; q)_{\infty}=\prod_{n=1}^{\infty}\left(1-q^{n}\right)=\sum_{n=-\infty}^{\infty}(-1)^{n} q^{n(3 n+1) / 2}$. The sum can be evaluated using the Poisson summation formula to yield 


$$
(q ; q)_{\infty}=\sqrt{\frac{2 \pi}{3 \gamma}} e^{\gamma / 24} \sum_{k=-\infty}^{\infty} e^{-\pi^{2} / 6 \gamma(2 k+1)^{2}-i \pi / 6(2 k+1)} .
$$

Again we use the fact that $\gamma \ll \pi^{2}$ and approximate the series by keeping only the $k=0$ and 1 terms. This gives $(q ; q)_{\infty}$ $\approx \sqrt{\frac{2 \pi}{\gamma}} q^{-1 / 24} e^{-\pi^{2} / 6 \gamma}$. This then leads to $K_{0}=1 / \pi$.

\section{RESULTS AND DISCUSSION}

In terms of the scaled variables $u=x / N^{\alpha}, v=y / N^{\alpha}$, the two-level kernel in the large $N$ limit is given by

$$
K^{P}(u, v ; \gamma)=\frac{1}{\pi} \frac{\sin \left[\frac{\pi}{\gamma} \ln \frac{u}{v}\right]}{u-v}, \quad 0<\gamma \ll \pi^{2}
$$

with density

$$
\sigma(u) \equiv K^{P}(u, u)=\frac{1}{\gamma u} .
$$

Thus the kernel is not universal and depends on one parameter that cannot be scaled out. Surprisingly, once unfolded by changing variables to

$$
\zeta=\frac{1}{\gamma} \ln u, \quad \eta=\frac{1}{\gamma} \ln v
$$

such that the density becomes unity, $\bar{\sigma}(\zeta)=1$, the scaled kernel becomes identical to the critical kernel of Eq. (5),

$$
\bar{K}^{P}(\zeta, \eta ; \gamma) \equiv \frac{K^{P}(\zeta, \eta ; \gamma)}{\sqrt{K^{P}(\zeta, \zeta ; \gamma) K^{P}(\eta, \eta ; \gamma)}}=K^{C}(\zeta, \eta ; \gamma)
$$

Thus it shows that rotationally invariant random matrix ensembles with inverse power law density $\sigma(x) \propto 1 / x$ belong to the class of critical ensembles, albeit after a logarithmic variable transformation.
It is important to emphasize that although the current model belongs to the same class of critical ensembles, it differs from the $N$-independent scaling model in two important ways. First, the $N$-dependent scaling introduces nontrivial $N$-dependent logarithmic potential in addition to the $\log$-squared potential of the $N$-independent scaling model. Second, the logarithmic variable transformation changes the interaction between the eigenvalues. It is therefore not obvious that the present model should lead to a critical ensemble. In the variable where both models have weakly confining $\log$-squared potential, the kernels for the $\mathrm{CB}$ scaling and the $\mathrm{N}$-independent scaling are given by Eqs. (24) and (5), respectively.

It has been conjectured before [20] that an inverse powerlaw density in a rotationally invariant random matrix ensemble approaches the same behavior as that for a weakly confined log-squared potential considered here. In this work we show explicitly by calculating exactly the two-level kernel that the inverse power case is a critical ensemble under the CB scaling. However, it differs from the case of the weakly confined log-squared potential with $N$-independent scaling in important ways; in the variable where the present kernel becomes identical to that for $N$-independent scaling, the confining potential is in fact Gaussian.

The Ismail-Masson polynomials go back to the classical orthogonal polynomials in the limit $q \rightarrow 1$. One can therefore hope to implement the scaling for the Ismail-Masson polynomials and then take the $q \rightarrow 1$ limit to obtain the corresponding scaling for the Gaussian ensembles. It turns out that the inverse power density $\sigma(u) \propto 1 / u$ is very special and exists only for $q<1$. The unfolded kernel with constant density of course has a well-defined $q \rightarrow 1$ limit which is just the sine kernel of the Gaussian ensemble. It should be possible to obtain the two-level kernel for arbitrary density power law with CB type scaling within our scheme, but it is not clear if a well-defined $N \rightarrow \infty$ limit exists for the resulting kernel. Here we presented only the inverse power density case, which we show to be critical.
[1] E. P. Wigner, Proc. Cambridge Philos. Soc. 47, 790 (1951); F. J. Dyson, J. Math. Phys. 3, 140 (1962).

[2] For recent reviews and applications see, T. Guhr, A. MuellerGroeling, and H. Weidenmueller, Phys. Rep. 299, 189 (1998); T. Guhr, A. Mueller-Groeling, and H. Weidenmueller, in Random Matrix Theory, edited by P. J. Forrester, N. C. Snaith, and J. J. M. Verbaarschot, special issue of J. Phys. A 36 (2003).

[3] M. L. Mehta, Random Matrices, 2nd ed. (Academic, New York, 1991).

[4] G. Szegö, Orthogonal Polynomials, 4th ed. (American Mathematical Society, Providence, 1975).

[5] P. J. Forrester, Nucl. Phys. B 402, 709 (1993); C. A. Tracy and H. Widom, Commun. Math. Phys. 159, 151 (1994).

[6] P. Cizeau and J. P. Bouchaud, Phys. Rev. E 50, 1810 (1994).

[7] K. A. Muttalib, Y. Chen, M. E. H. Ismail, and V. N. Nicopoulos, Phys. Rev. Lett. 71, 471 (1993); C. Blecken, Y. Chen, and K. A. Muttalib, in Symbolic Computation, Number Theory,
Special Functions, Physics and Combinatorics, edited by F. Garvan and M. Ismail (Kluwer Academic, Dordrecht, 2001).

[8] V. E. Kravtsov and K. A. Muttalib, Phys. Rev. Lett. 79, 1913 (1997); E. Bogomolny, O. Bohigas, and M. P. Pato, Phys. Rev. E 55, 6707 (1997); E. B. Bogomolny, U. Gerland, and C. Schmit, ibid. 59, R1315 (1999); A. M. Garcia-Garcia and J. J. M. Verbaarschot, ibid. 67, 046104 (2003).

[9] M. Moshe, H. Neuberger, and B. Shapiro, Phys. Rev. Lett. 73, 1497 (1994).

[10] A. D. Mirlin, Y. V. Fyodorov, F. M. Dittes, J. Quezada, and T. H. Seligman, Phys. Rev. E 54, 3221 (1996); S. M. Nishigaki, ibid. 59, 2853 (1999); A. D. Mirlin and F. Evers, Phys. Rev. B 62, 7920 (2000).

[11] L. Laloux, P. Cizeau, J. P. Bouchaud, and M. Potters, Phys. Rev. Lett. 83, 1467 (1999); A. Moustakas et al., Science 287, 287 (2000); M. Araujo, E. Medina, and E. Aponte, Phys. Rev. E 60, 3580 (1999). 
[12] Z. Burda, R. A. Janik, J. Jurkiewicz, M. A. Nowak, G. Papp, and I. Zahed, Phys. Rev. E 65, 021106 (2002).

[13] J. P. Bouchaud and A. Georges, Phys. Rep. 195, 127 (1990).

[14] J. Choi (unpublished).

[15] M. E. H. Ismail, Classical and Quantum Orthogonal Polynomials in One Variable (Cambridge University Press, Cambridge, England, 2005).

[16] M. E. H. Ismail and R. Zhang, Int. Math. Res. Notices 2006, 1 (2006).

[17] The asymptotic relation for the Ismail-Masson polynomials has been obtained in [16] for all $t>0$. Here we use only the result for $t<1 / 2$.

[18] E. T. Whittaker and G. N. Watson, A Course of Modern Analysis, 4th ed. (Cambridge University Press, Cambridge, England, 1927).

[19] See, e.g., G. Gasper and M. Rahman, Basic Hypergeometric Series (Cambridge University Press, Cambridge, England, 1990).

[20] A. C. Bertuola, O. Bohigas, and M. P. Pato, Phys. Rev. E 70, 065102(R) (2004). 